\title{
Distribution network management system: An AC OPF approach
}

DOI:

10.1109/PESMG.2013.6672895

Link to publication record in Manchester Research Explorer

\section{Citation for published version (APA):}

Alnaser, S. W., \& Ochoa, L. F. (2013). Distribution network management system: An AC OPF approach. In IEEE Power and Energy Society General Meeting/IEEE Power Energy Soc. Gen. Meet.

https://doi.org/10.1109/PESMG.2013.6672895

\section{Published in:}

IEEE Power and Energy Society General Meeting|IEEE Power Energy Soc. Gen. Meet.

\section{Citing this paper}

Please note that where the full-text provided on Manchester Research Explorer is the Author Accepted Manuscript or Proof version this may differ from the final Published version. If citing, it is advised that you check and use the publisher's definitive version.

\section{General rights}

Copyright and moral rights for the publications made accessible in the Research Explorer are retained by the authors and/or other copyright owners and it is a condition of accessing publications that users recognise and abide by the legal requirements associated with these rights.

\section{Takedown policy}

If you believe that this document breaches copyright please refer to the University of Manchester's Takedown Procedures [http://man.ac.uk/04Y6Bo] or contact uml.scholarlycommunications@manchester.ac.uk providing relevant details, so we can investigate your claim.

\section{OPEN ACCESS}




\title{
Distribution Network Mangement System:
}

\section{An AC OPF Approach}

\author{
Sahban W. Alnaser, Graduate Student Member, IEEE \\ Luis F. Ochoa, Senior Member, IEEE \\ School of Electrical and Electronic Engineering, The University of Manchester, Manchester, UK \\ sahban.alnaser@postgrad.manchester.ac.uk, luis_ochoa@ieee.org
}

\begin{abstract}
The transition towards low-carbon societies poses a significant paradigm shift to Distribution Network Operators (DNOs) around the world. The integration of low carbon technologies requires DNOs to move from semi-passive approaches to one where controllable devices and network participants are actively and optimally managed to ensure the adequate operation of the network. Here, it is proposed a distribution Network Management System (NMS) platform driven by an ac Optimal Power Flow (OPF) engine. The NMS is aimed to manage network voltages and congestion (thermal overloads) by coordinating the control of on-load tap changers, distributed generation reactive power and curtailment (as last resort). The proposed NMS is applied to a generic UK distribution network to assess its effectiveness in managing renewable DG considering minute by minute simulations. Results show the effectiveness of the technique in dealing with network constraints in real time.
\end{abstract}

Index Terms-- Active network management, distributed generation, distribution management system, generation curtailment, optimal power flow.

\section{INTRODUCTION}

Worldwide policies supporting the transition to a low carbon society require Distribution Network Operators (DNOs) to facilitate the adoption of low carbon technologies. However, the expected volumes of renewable Distributed Generation (DG), such as wind or solar power, pose significant technical (and economic) challenges. In particular, in a business as usual scenario, high volumes of wind power during minimum demand may result in voltages above the statutory limits as well as overload of assets [1]. DNOs traditionally avoid these issues by either limiting the installed DG capacity or upgrading the corresponding assets. In the $\mathrm{UK}$, to allow the connection of larger volumes of DG, nonfirm connection agreements are approved in which DNOs can disconnect DG plants if issues arise. However, the latter approach may reduce the potential energy from renewable sources and may increase the frequency of disconnections for some DG plants. Consequently, a more intelligent management of the network is required.

In the last few years, only a few UK DNOs have implemented Active Network Management (ANM) schemes to deal with constraints in real time. An example of this is the application of wind power curtailment to single or multiple sites and reduce partially their power production to reach acceptable network operating margins (i.e., thermal and voltage limits) [2].To increase further the harvesting of low- carbon electricity, reactive power capabilities of modern wind farms could also be used to alleviate voltage rise problems during high wind power injections and light demand [3]. Moreover, active management of on-load tap changers (OLTC) at substations could also allow alleviating voltage issues, thus minimizing curtailment [4].

Advanced or future Distribution Management System (DMS) algorithms have been proposed in the literature to achieve different objectives. In [5], a rule based decision making (heuristic) is proposed to maintain voltage within statutory limits whilst increasing the harvesting from DG plants. In [6], a dc Optimal Power Flow (OPF) is applied to minimize curtailment of DG required to decongest the network. This power flow management is triggered once the thermal constraints in a number of observable corridors are violated. These two DMS approaches do not provide complete, integrated solutions for both thermal overloads and voltage excursion problems. In [7], a DMS is proposed to tackle both constraints. Coordination between DG curtailment processes and voltage control devices is adopted to minimize energy cost. However, the optimization problem is formulated in a simplified manner using linear programming and voltage sensitivity coefficients. The above studies, except from [6], do not consider the time-series nature of wind and demand, which is necessary to adequately model real-time operation.

To accelerate the transition to a low carbon economy networks need to be managed actively. However, DG curtailment should be restricted as much as possible. In this work, it is proposed a comprehensive distribution Network Management System (NMS) aimed at managing network voltages and congestion (thermal overloads) through the intelligent, coordinated control of OLTCs, DG reactive power output and generation curtailment (as last resort). Here, a centralized ac OPF (i.e., a non-linear programming problem) tailored to produce the optimal set points for the active elements is adopted [4, 8-9].

This paper is structured as follows: Section II presents the architecture of the distribution NMS. The formulation of the optimizer engine in the NMS is explained in section III. In section IV the application of the proposed NMS on a generic UK distribution network incorporating multiple wind farms is illustrated. Finally, the conclusions are drawn in section V.

\section{ARCHITECTURE OF THE DISTRIBUTION NMS}

The proposed distribution NMS platform is shown in Fig. 1. Pseudo real-time measurements, to be in practice managed by a SCADA system, are obtained from time-series 
simulations run by a distribution network modeled in OpenDSS [10]. Network elements are monitored in order to identify branches (i.e., lines or transformers) exceeding their thermal capacities or nodes with voltages beyond the statutory limits. The NMS ac OPF-based optimization engine, implemented in the modeling language AIMMS [11], finds the best set points for the available controllable resources (i.e., OLTCs, DG power factor, and DG curtailment) upon the violation of constraints (or the existence of curtailment).

\section{PROPOSED AC OPF-BASED NMS}

While SCADA systems can provide true real-time monitoring of network elements (or participants), in distribution networks this is typically limited to minutes (e.g., 30 minutes). In order to assess the benefits of higher sampling rates, the proposed NMS considers much smaller time intervals in order to closely follow the network behavior due to variations in -particularly- generation.

Here, the time interval between two consecutive constraint checks is defined as control cycle. At the end of each control cycle, if a constraint is violated (or control actions have taken place and can now return to normal operation) the NMS optimization engine will produce the best set points for the operation of voltage control devices (i.e., OLTCs at substations) and the reactive power output of DG plants in such a way that curtailment is considered as the last resort. These new set points will be used throughout the following control cycle. Note that the NMS should comply with the existing connection agreements that might allow some DG plants to deliver power up to its rated capacity (i.e., firm connections).

Let us consider two sets of DG plants: $G$ (indexed by $g$ ), with firm connections, and $N$ (indexed by $n$ ), with controllable connections. The corresponding instantaneous (i.e., at the control cycle) active and reactive power outputs are denoted by $p_{g}, p_{n}$ and $q_{g}, q_{n}$, respectively. As a proxy of minimizing DG curtailment, the ac OPF formulation has as objective to maximize the total active power of the controllable DG plants:

$$
\max \sum_{n \in N} p_{n}
$$

The objective function will be subject to a range of constraints. The maximum power that can be extracted from each DG plant is restricted by the available generation level $p_{n}^{+}$at the time of triggering the controller:

$$
p_{n} \leq p_{n}^{+} \quad \forall n \in N
$$

Voltage magnitude at bus $b$ ( $B$, set of buses) should be maintained within the statutory limits $V_{b}^{(+,-)}$.

$$
V_{b}^{-} \leq V_{b} \leq V_{b}^{+} \quad \forall b \in B
$$

The apparent power flow at the start and the end of lines and transformers, $l$ ( $L$, set of lines) are limited to the corresponding capacity, as formulated in (4).

$$
\left(f_{l}^{(1,2), P}\right)^{2}+\left(f_{l}^{(1,2), Q}\right)^{2} \leq\left(f_{l}^{+}\right)^{2} \quad \forall l \in L
$$

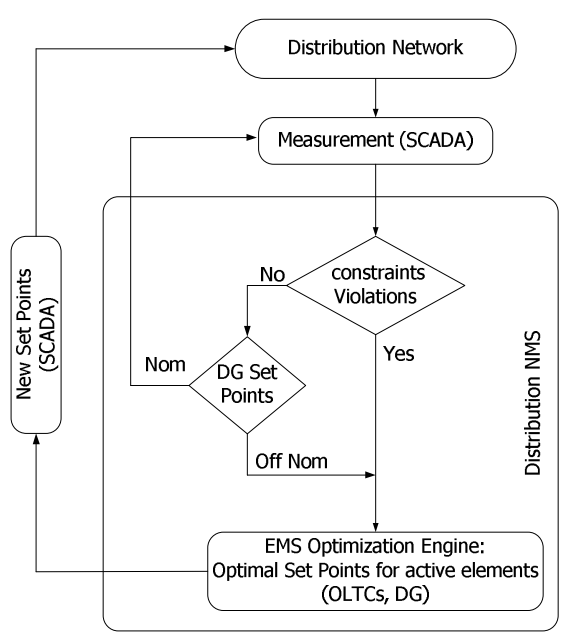

Figure 1. Architecture of the proposed Distribution NMS

where $f_{l}^{(1,2), P}$ and $f_{l}^{(1,2), Q}$ are active and reactive power into the start bus and the end bus of the element $l$ (denoted by 1 and 2), respectively, and $f_{l}^{+}$is the thermal capacity of $l$.

Active and reactive power injections onto the start and end bus for each line and each transformer, are calculated according to Kirchhoff's Voltage Law (KVL) in (5) and (6), respectively:

$$
\begin{array}{ll}
f_{l}^{(1,2),(P)}=f_{l, K V L}^{(1,2),(P)}(V, \delta) & \forall l \in L \\
f_{l}^{(1,2),(Q)}=f_{l, K V L}^{(1,2),(Q)}(V, \delta) & \forall l \in L
\end{array}
$$

where $f_{l, K V L}^{(1,2),(P)}(V, \delta)$ and $f_{l, K V L}^{(1,2),(Q)}(V, \delta)$ are the standard KVL expressions.

If the transformers are equipped with voltage regulation devices that are remotely controlled by the DMS, the corresponding terms in (5) and (6) for voltage at the start bus of the line will be divided by the tap ratio $\tau_{l},\left(\tau_{l}^{-} \leq \tau_{l} \leq \tau_{l}^{+}\right)$. However, given that the ac OPF is formulated as a non-linear programming problem (i.e., continuous variables), the tap ratio requires a post-optimization adjustment. It should be converted upwards to the nearest discrete value that reflects a feasible position $m_{l} \in\left\{M^{-}, \ldots,-1,0,1, \ldots, M^{+}\right\}$, where $M^{(-,+)}$ represents the minimum and maximum tap positions. This approximation is applied in (7):

$$
m_{l}=\left\lceil\left(\tau_{l}-1\right) / R_{l}\right\rceil
$$

where $R_{l}$ is the size of regulation step as in (8):

$$
R_{l}=\left(\tau_{l}^{+}-\tau_{l}^{-}\right) /\left(M^{+}-M^{-}\right)
$$

The tap positions prior to the NMS control action could be provided to the OPF to increase the speed of the optimization (this is outside the scope of this work). For those OLTCs locally controlled with respect to the measurements at the regulated terminal, the values of the tap positions can be included in the optimization engine as fixed parameters.

Controllable DG units can also operate at different power factors, making reactive power "dispatchable". To incorporate this into the ac OPF framework, the power factor angle for 


\section{Accepted Paper}

each controlled DG, $\emptyset_{n}$, is considered as a variable and it is required to operate within the DG plant's capability:

$$
\emptyset_{n}^{-} \leq \emptyset_{n} \leq \emptyset_{n}^{+} \quad \forall n \in N
$$

Kirchhoff's Current Law describes the conservation of real and reactive power at bus $b, \forall b \in B$ according to (10) and (11), respectively:

$$
\begin{gathered}
\sum_{n \in N \mid \beta_{n=b}} p_{n}+\sum_{g \in G \mid \beta_{g=b}} p_{g}+\sum_{x \in X \mid \beta_{x=b}} p_{x} \\
=d_{b}^{P}+\sum_{l \in L \mid \beta_{l}^{(1,2)}=b} f_{l}^{(1,2),(P)} \\
\sum_{n \in N \mid \beta_{n=b}} p_{n} \tan \left(\varnothing_{n}\right)+\sum_{g \in G \mid \beta_{g=b}} q_{g}+\sum_{x \in X \mid \beta_{x=b}} q_{x} \\
=d_{b}^{Q}+\sum_{l \in L \mid \beta_{l}^{(1,2)}=b} f_{l}^{(1,2),(Q)}
\end{gathered}
$$

where $\beta_{m}$ maps the location of each network element ( $m \subset$ $\{n, g, x, l\})$ to its associated bus and $d_{b}^{(P, Q)}$ are the active and reactive demand at the same bus. The distribution network will be supplied by at least one point interfacing with the upstream grid. It is assumed that this point $x$ ( $X$, set of external connections) can import/export real and reactive power $\left(p_{x}, q_{x}\right)$.

The set points, $C S_{n}$, for the controllable DG plants in the next control cycle are calculated using (12) with the assumption that demand and the. DG primary resource (e.g., wind) will both remain constant (i.e., persistence forecasting).

$$
C S_{n}=p_{n} / p_{n}^{+} \quad \forall n \in N
$$

\section{CASE STUdy: UKGDS EHV1 NeTWORK}

The proposed distribution NMS will be applied to a generic UK distribution network in order to assess its effectiveness in managing renewable DG with realistic control cycles. Fig. 2 shows the EHV1 network from the UK Generic Distribution System (GDS) [12]. The 33kV-feeders are supplied by two identical 30 MVA $132 / 33 \mathrm{kV}$ transformers (BSP stands for bulk supply point). The voltage of $33 \mathrm{kV}$ system is regulated by the OLTC at the $132 \mathrm{kV} / 33 \mathrm{kV}$ substation and a voltage regulator located between buses 304 and 321. Similarly, all primary substations $(33 \mathrm{kV} / 11 \mathrm{kV})$ are equipped with OLTCs to regulate the voltage to a pre-defined target of 1.0 p.u. Here, the adopted regulation range for all OLTCs as well as the voltage regulator is $-15 \%$ to $+5 \%$ in 16 steps of $1.25 \%$ (using a deadband of $\pm 1 \%$ for those locally controlled). In the UK, the statutory limits for these distribution voltages ( $11 \mathrm{kV}$ to $33 \mathrm{kV}$ ) are $\pm 6 \%$ of nominal.

Three wind farms with a total capacity of $25.3 \mathrm{MW}$ are connected to the network as shown in Fig. 2 (values found in in [4]). These generators will have the same wind resource as they are located in a small geographical area (an island, hence the subsea cable). All the generators are capable of operating with power factors between 0.95 inductive and capacitive according to UK requirements [13]. In addition, the power factor setting is considered to be dispatchable, i.e., remotely

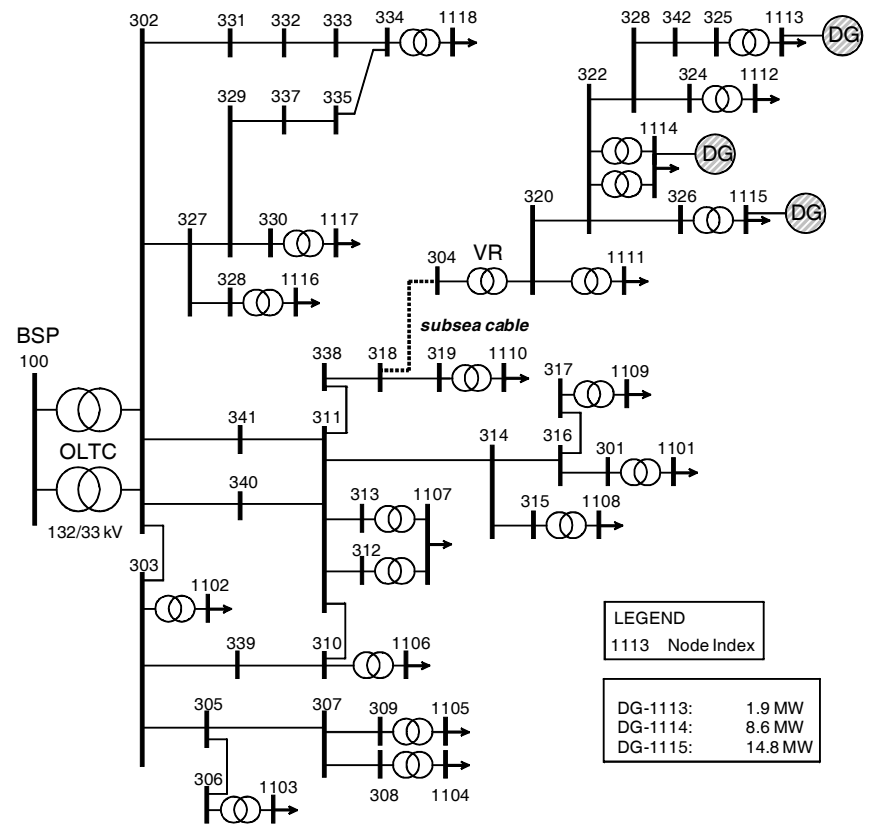

Figure 2. UK GDS EHV1 Network [12] and adopted capacities of DG.

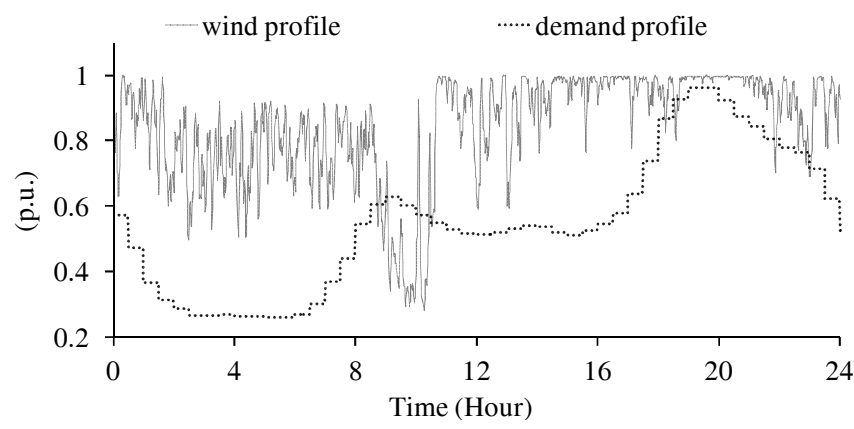

Figure 3. Normalized wind and demand profiles $\left(1^{\text {st }}\right.$ February 2010$)$.

controllable. The demand and wind profiles for $1^{\text {st }}$ February 2010 are shown in Fig. 3. The maximum and minimum demands of the island are 6.36 and $1.5 \mathrm{MW}$, respectively. A normalized half-hourly load profile for UK 'domestic unrestricted' customers for the North West of England was adopted.

To examine the benefits from the proposed distribution NMS in managing network constraints, the business as usual operation (i.e., no active management) is compared with different active network solutions. Given that simulations are carried out with one minute resolution, the analyzed period is limited to one day of the first week of February 2010.

\section{A. Business as Usual Operation}

This case is only meant to show the potential voltage and thermal issues that might arise if the total wind power capacity of 25.3 MW is connected without any form of control. In practice, the corresponding DNO would not allow to connect more than circa 16.5 MW based on the constraints during the min demand and max generation scenario for the island.

Indeed, it is particularly during this worst case scenario that the wind farms will create a significant reverse power 


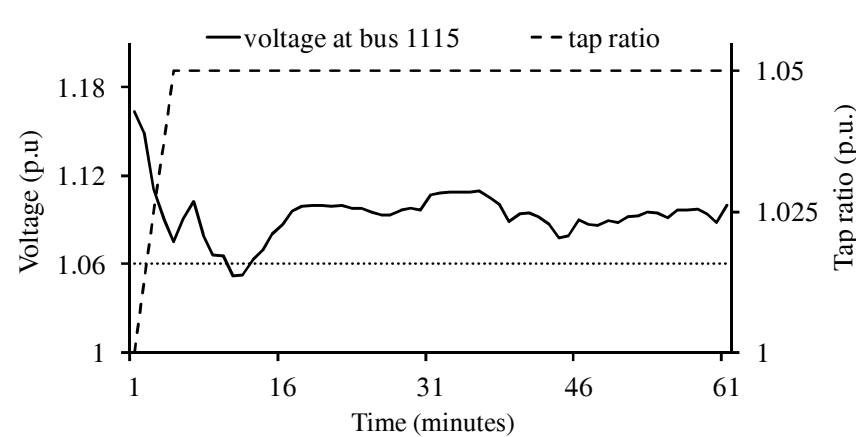

Figure 4. Business as Usual Operation: Voltage profile p.u. at bus 1115 and tap ratio at transformer 326-1115.

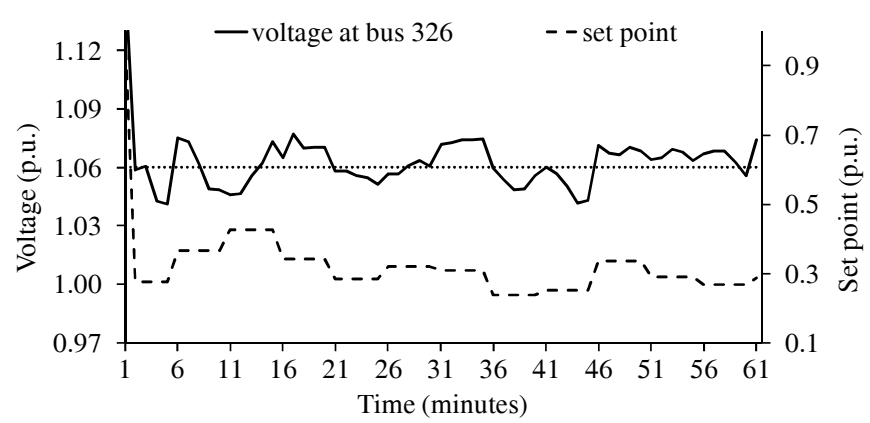

Figure 5. Curtailment-only NMS: Voltage profile at bus 326 and set point for the wind farm at bus 1115 .

flow through the lines (338-318, 318-304 and 320-322) as well as the voltage regulator. This would exceed their thermal limits (15 MVA) by around $40 \%$. In addition, the voltage at most buses in the island would also exceed the upper limit. The highest value (1.17 p.u.) occurs at bus 326 that shows voltage excursions during $36 \%$ of the analyzed week. This is a consequence of the limitations of voltage regulation devices such as the OLTC of the transformer 326-1115 as shown in Fig. 4. However, the OLTCs that regulate the load buses 1112, 1113 and 1114 can actually handle the corresponding voltage excursions given that these are individual DG sites.

\section{B. Curtailment-only NMS}

Here, the proposed ac OPF-based distribution NMS is continuously monitoring network elements and controlling (if necessary) the output of DG plants every five minutes, i.e., a control cycle of five minutes. Fig. 5 illustrates the operation of the curtailment-only NMS over one hour for the wind farm at bus 1115 . It can be seen that in minute 1 , the controller is activated to solve the voltage rise at bus 326 . Based on the optimisation engine, the NMS sends a control signal to the wind farm in order to pitch the turbines' blades in a way that the maximum capacity is now $28 \%$ of nominal. Although this setting will be kept until the next control cycle, the one-minute resolution simulations show the corresponding response. At minute 5 there is no constraint violation. However, given that curtailment has been applied to the wind farm, the NMS will trigger the optimization to find a new set point. It can be seen that it is indeed partially improved at minute 6 (to 37\%). This process of monitoring and updating the wind farm's set point accordingly is carried out at each control cycle. Furthermore, it can be observed that at minute 45 , the optimizer decides to raise the wind farm's set point based on the voltage (within
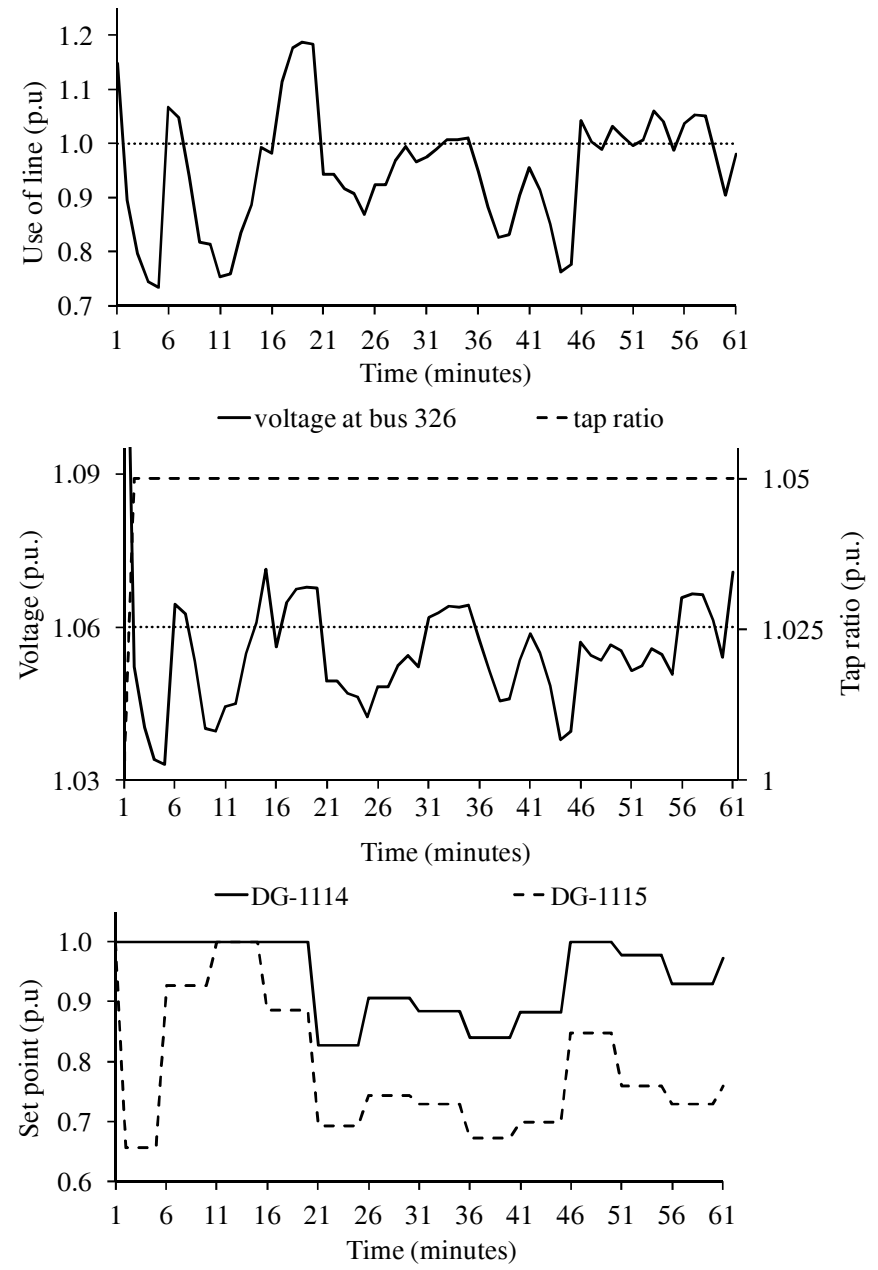

Figure 6. Curtailment and CVC NMS: (Top) Use of line 320-322 p.u.; (middle) Voltage profile at bus 326 p.u. and tap ratio at the voltage regulator; and (bottom) set points of DG at buses 1114 and 1115.

limits). Nonetheless, in the next minute the wind speed increases resulting in voltage rise beyond the limit. This (minor) error is due to the adopted persistence forecasting model that considers that the wind resource and demand remain the same from one cycle to the next one. However, the use of short control cycles (such as 5 minutes) allow closely following wind variability and most likely preventing the impacts of substantial changes in generation.

In this case study, it is clear that voltage is the binding constraint that drives curtailment of the wind farm at bus 1115. Since curtailment is significant, potential thermal overloads at all upstream lines are prevented as a result.

\section{Curtailment and Coordinated Voltage Control NMS}

The coordinated voltage control (CVC) of the substations and voltage regulators, as initially presented in [4], can alleviate voltage excursions and decrease the use of curtailment. Indeed, given that objective function adopted for the proposed optimization engine, controllable voltage regulation devices will be first auctioned leaving curtailment as the last resort. The loading of line 320-322, the voltage profile at bus 326 , and the set points of two wind farms are all shown in Fig. 6 for the same hour in February. It can be seen 


\section{Accepted Paper}
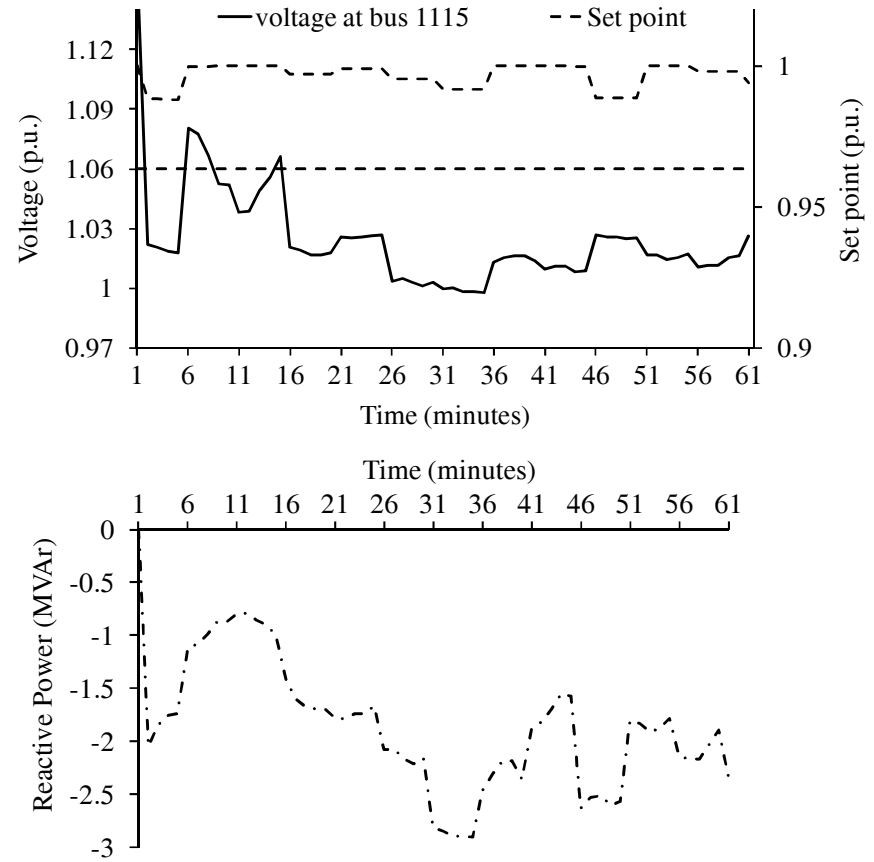

Figure 7. (Top) Voltage profile at bus 326 p.u. and set point for DG at bus 1115, with CVC and PFc, and (bottom) reactive power for DG 1115.

that after minute 1 , the NMS manages the corresponding thermal and voltage constraints. For instance, in minute 20, due to a wind gust, the use of the line exceeds its capacity by $18 \%$ and the voltage at bus 326 goes beyond the statutory limit (albeit within the control deadband). Thus, the NMS reacts adjusting the set points of the voltage regulator and the wind farms (1114 and 1115). The wind farm at bus 1113 is not affected. Crucially, the one at 1115 is given a set point of $69 \%$, allowing more power injection than when only applying curtailment (28\%). This is possible given that the taps of the voltage regulator are increased to help managing bus 326.

\section{Curtailment, CVC and Power Factor Control NMS}

By also controlling the power factor of wind farms it is possible to avoid further curtailment. In this case, at each time the optimization engine is triggered, it will also find the best power factor setting that minimizes the amount of curtailment. Note that if the controller opts to use an inductive power factor to manage voltages, the complex power flows through the lines might increase depending on the wind resource after the control action. Thus, to avoid potential congestion issues, the power factor capability is limited to 0.98 inductive/capacitive. In Fig. 7, the wind farm at bus 1115 operates at inductive power factor (mostly close to 0.98 ) to mitigate voltage issue and support the CVC in order to minimize curtailment. This voltage profile is maintained within the statutory limits and, consequently, the wind farm's set point is most of the time close to unity. The variation of the reactive power follows the active power of the wind resource (based on the power factor).

\section{E. Energy Harvesting}

Although it is not the main interest of DNOs in countries with unbundling rules, the energy harvested by the DG plants can be used as a metric for the NMS. For the first week of February 2010, the application of the full NMS (section IV.D) leads to $1.45 \mathrm{GWh}$ of production, which is $28 \%$ above the curtailment scheme alone (section IV.B).

\section{CONCLUSIONS}

An ac OPF-based distribution NMS was proposed that allows actively managing network voltages and congestion in real-time operation whilst minimizing DG curtailed power. This is achieved through optimal coordination of voltage control devices and DG power factor, using as last resort DG curtailment. The proposed NMS was applied to a generic UK distribution network for one week using wind and load profile with one-minute resolution. The results show that the proposed NMS is capable of managing the network within voltage and thermal capacity limits whilst minimizing DG curtailment.

Future work will cover the impact of varying the control cycle length to monitor the network status periodically and follow the variability in wind resource. The proposed framework will also be further developed to incorporate future network devices (e.g., energy storage) and demand-based approaches (e.g., demand side management, voltage-sensitive models of load, etc.).

\section{REFERENCES}

[1] R. A. Walling, R. Saint, R. C. Dugan, J. Burke, and L. A. Kojovic, "Summary of Distributed Resources Impact on Power Delivery Systems," IEEE Transactions on Power Delivery, vol. 23 no. 3 pp. 1636 - 1644 July 2008.

[2] R. A. F. Currie, G. W. Ault, C. E. T. Foote, and J. R. McDonald, "Active power-flow management utilising operating margins for the increased connection of distributed generation," IET Proceedings Generation, Transmission \& Distribution, vol. 1, no. 1, pp. 197-202, Jan. 2007.

[3] L. F. Ochoa, A. Keane, and G. P. Harrison, "Minimizing the reactive support for distributed generation: Enhanced passive operation and smart distribution networks," IEEE Trans. on Power Systems, vol. 26, no. 4, pp. 2134-2142, Nov. 2011.

[4] L. F. Ochoa, C. Dent, and G. P. Harrison, "Distribution network capacity assessment: Variable DG and active networks," IEEE Trans. on Power Systems, vol. 25, no. 1, pp. 87-95, Feb. 2010.

[5] T. Xu, N. S. Wade, E. M. Davidson, P. C. Taylor, S. D. J. McArthur, and W. G. Garlick, "Case-Based Reasoning for Coordinated Voltage Control on Distribution Networks," Electric Power Systems Research vol. 81, no. 12, pp. 2088-2098, December 2011.

[6] M. J. Dolan, E. M. Davidson, I. Kockar, G. W. Ault, McArthur, and S.D.J., "Distribution Power Flow Management Utilizing an Online Optimal Power Flow Technique "IEEE Transactions on Power Systems, vol. 27, no. 2, pp. 790-799, May 2012.

[7] F. Pilo, G. Pisano, and G. G. Soma, "Optimal Coordination of Energy Resources With a Two-Stage Online Active Management " IEEE Transactions on Industrial Electronics, vol. 58, no. 10, pp. 4526 - 4537 Oct. 2011.

[8] C. Dent, L. F. Ochoa, G. P. Harrison, and J. W. Bialek, "Efficient secure AC OPF for network generation capacity assessment," IEEE Trans. on Power Systems, vol. 25, no. 1, pp. 575-583, Feb. 2010.

[9] L. F. Ochoa and G. P. Harrison, "Using AC optimal power flow for DG planning and optimisation," in Proc. 2010 IEEE/PES General Meeting, pp. 7.

[10] Open Distribution System Simulator - OpenDSS. [Online]. Available: http://sourceforge.net/apps/mediawiki/electricdss

[11] J. Bisschop and M. Roelofs, "AIMMS - The user's guide," P. D. Technology., Ed., 2012.

[12] Distributed Generation and Sustainable Electrical Energy Centre. United Kingdom Generic Distribution System (UK GDS). [Online]. Available: http://www.sedg.ac.uk/

[13] National Grid Electricity Transmission, The grid code, Aug. 2012. 